\title{
Repetitive negative thinking in daily life and functional connectivity among default mode, fronto-parietal, and salience networks
}

\author{
D. M. Lydon-Staley $\mathbb{1}^{1}$, C. Kuehner $\mathbb{D}^{2}$, V. Zamoscik $\mathbb{B}^{3}$, S. Huffziger, ${ }^{2,4}$, P. Kirsch $\mathbb{B}^{3}$ and D. S. Bassett $\mathbb{D}^{1,5,6,7,8,9}$
}

\begin{abstract}
Repetitive negative thinking (RNT) is a maladaptive response to sadness and a transdiagnostic risk-factor. A critical challenge hampering attempts to promote more adaptive responses to sadness is that the between-person characteristics associated with the tendency for RNT remain uncharacterized. From the perspective of the impaired disengagement hypothesis, we examine between-person differences in blood-oxygen-level-dependent (BOLD) functional networks underlying cognitive conflict signaling, self-referential thought, and cognitive flexibility, and the association between sadness and RNT in daily life. We pair functional magnetic resonance imaging with ambulatory assessments deployed 10 times per day over 4 consecutive days measuring momentary sadness and RNT from 58 participants ( 40 female, mean age $=36.69$ years; 29 remitted from a lifetime episode of Major Depression) in a multilevel model. We show that RNT increases following sadness for participants with higher than average betweennetwork connectivity of the default mode network and the fronto-parietal network. We also show that RNT increases following increases in sadness for participants with lower than average between-network connectivity of the frontoparietal network and the salience network. We also find that flexibility of the salience network's pattern of connections with brain regions is protective against increases in RNT following sadness. Our findings highlight the importance of functional brain networks implicated in cognitive conflict signaling, self-referential thought, and cognitive flexibility for understanding maladaptive responses to sadness in daily life and provide support for the impaired disengagement hypothesis of RNT.
\end{abstract}

\section{Introduction}

The experience of sadness is functional as it highlights a discrepancy between one's actual state and one's desired state $^{1}$. Cognitive responses to sadness, however, do not always support well-being. Repetitive negative thinking (RNT) entails perseverative thinking about one's problems or emotions and is a maladaptive response to sadness $^{2-4}$ and a transdiagnostic risk factor for psychopathology $^{5-11}$. The impaired disengagement hypothesis ${ }^{12}$

\footnotetext{
Correspondence: D. S. Bassett (dsb@sas.upenn.edu)

${ }^{1}$ Department of Bioengineering, School of Engineering and Applied Sciences, University of Pennsylvania, Philadelphia, PA 19104, USA

${ }^{2}$ Research Group Longitudinal and Intervention Research, Department of Psychiatry and Psychotherapy, Central Institute of Mental Health, Medical Faculty Mannheim, Heidelberg University, 68159 Mannheim, Germany

Full list of author information is available at the end of the article.
}

proposes that enduring negative thoughts, especially those directed towards the self, signal a cognitive conflict that leads to the disengagement of attention from negative thoughts via attentional control. From this perspective, RNT results from impaired cognitive conflict signaling and/or difficulties in enacting attentional control to divert attention away from one's negative thoughts. In line with this perspective, deficits in inhibiting previous mental states are associated with $\mathrm{RNT}^{13}$ and cognitive control ability moderates the extent to which RNT follows negative moods ${ }^{14}$.

Three large-scale functional brain networks are increasingly incorporated into the impaired disengagement hypothesis due to their roles in conflict signaling, self-referential thought, and attentional control ${ }^{12,15}$. The 
default mode network (DMN) is characterized by a tendency to deactivate during tasks and to activate at rest, as well as during self-referential tasks ${ }^{16,17}$. The frontoparietal network (FPN) is comprised of regions that have established roles in attentional control and working memory ${ }^{18-20}$, response selection ${ }^{21}$, and response suppression $^{22}$. Due to its role in top-down, executive functions, the FPN is viewed as essential for guiding flexible, goal-directed behavior. Connectivity among the FPN and DMN provides important information about the capacity for flexible behavior, such as attentional control, with greater strength of connectivity between the DMN and the FPN associated with poorer cognitive task performance $^{23,24}$. The salience network (SN) has two primary functions (for reviews, see refs. ${ }^{25,26}$ ). One function relates to salience detection and the second relates to the facilitation of access to cognitive control resources (e.g., attention, working memory) following the detection of salient stimuli. The access to cognitive control is facilitated by signaling the engagement of the FPN while suppressing DMN activity ${ }^{15,27}$.

Alterations in DMN functioning in depression (see refs. ${ }^{28,29}$ for reviews) are theorized to reflect impairments in switching between internally and externally directed thought that lead to RNT tendencies. Less work has focused on the DMN's role in RNT in relation to both the FPN and the SN (see ref. ${ }^{30}$ for review). A notable exception observed that increasing dominance of DMN activity over FPN activity is associated with increasing levels of depressive rumination ${ }^{31}$. Furthermore, differences emerge in the timing of activity in the insula (regions of the SN) prior to increases in FPN activity (and decreases in DMN activity) in depressed individuals relative to healthy controls, indicating a role for the $\mathrm{SN}$ in coordinating DMN and FPN activity in RNT (see also ref. ${ }^{32}$ ).

We examine RNT as it occurs in response to sadness during the course of daily life in 29 remitted depressed individuals and 29 matched healthy controls. The inclusion of remitted depressed individuals ensured adequate between-person variability in sad mood and RNT, given findings that levels of RNT and negative mood are elevated in remitted depression ${ }^{33}$. Based on the role of the SN in switching between DMN and FPN activity upon the identification of cognitive conflict, we hypothesize that greater connectivity among the $\mathrm{SN}$ and the FPN, and among the $\mathrm{SN}$ and the DMN is associated with reduced RNT tendencies. In addition, given evidence that increased connectivity among the FPN and the DMN is associated with reduced ability to deploy cognitive functions, we hypothesize that increased connectivity among the FPN and the DMN is associated with a greater propensity to respond to sadness with RNT. We also test the moderating role of $\mathrm{SN}$ flexibility on the association between sadness and RNT. The SN is a uniquely flexible system, exhibiting substantial time-varying functional interactions with other functional networks ${ }^{34,35}$ and greater SN flexibility is associated with greater cognitive flexibility $^{34}$. Based on the association between SN flexibility and cognitive flexibility, we hypothesize that greater SN flexibility is associated with a reduced tendency to follow sadness with RNT.

\section{Materials and methods}

We made use of data from a study designed to provide insight into cognitive and affective function in individuals with remitted major depressive disorder. Detailed information on the larger study is available in refs. ${ }^{36-39}$. The datasets analyzed and code used during the current study are available from the corresponding author upon reasonable request. The study was approved by the local ethics committee of the University of Heidelberg and conformed to the Declaration of Helsinki.

\section{Participants}

Participants were 29 remitted depressed individuals with $\geq 2$ episodes of major depressive disorder and 29 age-, sex-, and education-matched healthy controls without current or lifetime diagnosis for major depressive disorder. Participants were recruited by announcements in local newspapers and on the homepage of the Central Institute of Mental Health (CIMH), Mannheim, Germany. The remitted depressed individuals had to be in partial or full remission for at least two months. Exclusion criteria for all participants included clinical diagnoses of bipolar and psychotic disorders, substance dependence, current substance abuse, generalized anxiety disorder, current obsessive-compulsive, post-traumatic stress, and eating disorders according to DSM-IV as well as contraindications for magnetic resonance imaging. Psychopathology-related criteria for inclusion were assessed by a trained clinical psychologist who used the Structured Clinical Interview for DSM-IV axis $\mathrm{I}^{40}$. Detailed information about the sample used for the analyses is presented in Supplementary Table 1. The study was approved by the local ethics committee of the University of Heidelberg. All participants gave informed consent in writing.

\section{Procedure}

We show a schematic of the procedure in Fig. 1. Demographic and clinical variables were collected at a baseline session. Affective and cognitive state variables were then measured by ambulatory assessment ${ }^{41}$. Ambulatory assessment was completed over four consecutive weekdays with 10 assessments per day using smartphones (HTC Touch Diamond 2) and the software MyExperienceIDE by movisens $\mathrm{GmbH}$ (Karlsruhe, 


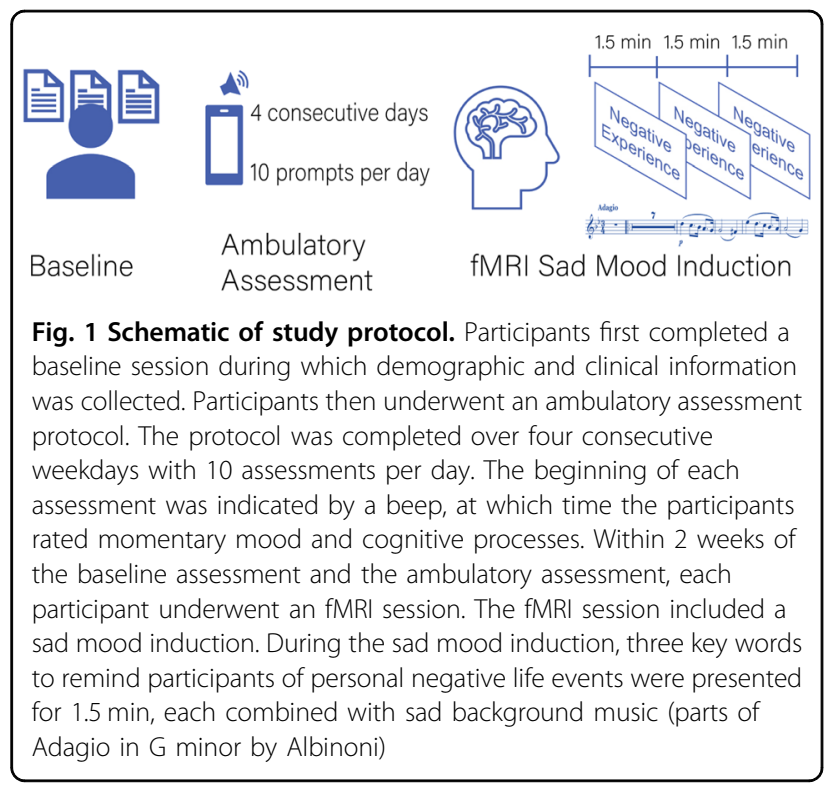

Germany). The beginning of each assessment was indicated by a beep, at which point the participants rated momentary mood and cognitive processes. The analyses in the present study focused on reports of sadness and RNT at 10 assessments on each of the four days (a potential total of 40 assessments per person) during which participants reported on their levels of sadness and also their RNT.

The fMRI session took place within the two week period following the baseline assessment and the ambulatory assessment. Each participant underwent a sad mood induction (4.5 $\mathrm{min}$ ) and further tasks which are not subject to the following analyses. During the sad mood induction, three key words were presented to remind participants of personal negative life events. The key words were presented for $1.5 \mathrm{~min}$ each during the sad mood induction scan, were chosen by participants, and were related to three negative life events produced after participants were individually assessed immediately prior to the fMRI session. Participants were also presented with sad background music (Adagio in $\mathrm{G}$ minor by Albinoni) during the sad mood induction scan.

\section{Measures}

We made use of demographic and depressive symptom data collected during the baseline session, fMRI data collected during the sad mood induction scan, and sadness and RNT data from the ambulatory assessment.

\section{Momentary sadness}

Sadness was assessed during the ambulatory assessment protocol with the item "At the moment I feel sad", rated on a scale from 0 (not at all) to 6 (very much).

\section{Momentary repetitive negative thinking}

$\mathrm{RNT}^{42}$ was assessed during the ambulatory assessment protocol with the item "At the moment, I am stuck on negative thoughts and cannot disengage from them", rated on a scale from 0 ("not at all") to 7 ("very much"). This measure has been used previously in ambulatory assessment studies of rumination ${ }^{37,43}$.

\section{Depressive symptoms}

At baseline, depressive symptoms during the previous 2 weeks were assessed with the self-rated Beck Depression Inventory II-Revised (BDI II ${ }^{44}$; German version ${ }^{45}$ ) and the Montgomery and Asberg Depression Rating Scale (MADRS $^{46}$; German version ${ }^{47}$ ) rated by a trained clinical psychologist, both of which have shown good reliability, validity, and sensitivity to symptom changes ${ }^{48}$. We created a composite score for depressive symptoms by averaging the z-standardized BDI-II and MADRS scores ${ }^{36,49}$.

\section{Blood-oxygen-level-dependent (BOLD) functional connectiv- ity networks}

A summary of our analysis of the functional imaging data is as follows: BOLD time series from the sad mood induction phase were preprocessed and an association matrix representing the functional connectivity among regions of the brain was created before connectivity indices of cognitive control were created. Additional detail is provided below.

Data acquisition One-hundred and eighty $\mathrm{T} 2 \%$ weighted EPI images (TR $=1.5 \mathrm{~s}, \alpha=80^{\circ}, \mathrm{TE}=28 \mathrm{~ms}$ ) with 24 slices (slice thickness $4 \mathrm{~mm}$, voxel size $3 \times 3 \times 4 \mathrm{~mm}^{3}$, FOV $192 \mathrm{~mm}$ ) were recorded with a $3 \mathrm{~T}$ TIM Trio Scanner with a 12 channel head coil (Siemens Medical Systems, Erlangen, Germany). To allow participants to adapt to the scanner environment and to reduce between-volume variance, the first 20 images of each measurement were discarded.

Data preprocessing We processed the fMRI data with a preprocessing scheme based on studies that evaluated the performance of a wide variety of preprocessing pipelines in mitigating motion artifact in studies of BOLD functional connectivity ${ }^{50,51}$. The functional data underwent de-spiking to smooth outliers in each voxel using AFNI's $^{52}$ 3dDespike, rigid transformation to correct for head motion using FSL's ${ }^{53}$ MCFLIRT, and slice-time correction using FSL Slicetimer to control for temporal differences in the order of the acquisition of the slices in each brain volume. Skull stripping was performed on the structural data using FSL BET. The mean functional image was computed and bias-corrected, and the skullstripped functional image was bias-corrected. Advanced normalization tools $\left(\mathrm{ANTs}^{54}\right)$ were used to compute the 
transformation parameters for the mean functional image to the high-resolution structural image. ANTs segmentation was performed to obtain a warped structural image, a skull-stripped brain mask, and masks for white matter and cerebrospinal fluid. Confound regression was then conducted. The time series was detrended by regressing the time series on the mean and the polynomial trends, up to quadratic terms. AFNI's 3dbandpass was used to filter out very high or very low fluctuations in the signal (with a high pass of 0.01 and a low pass of 0.12). Six head motion regressors and three matter regressors (global signal, white matter, and cerebrospinal fluid), as well as their derivatives, quadratic terms, and the squares of their derivatives (36 regressors in total) were regressed from the time series. ANTs was used to warp the high-resolution structural image to the MNI template. The transformation parameters from the ANTs functional to structural co-registration and the transformation parameters from the ANTs structural to MNI co-registration were used to warp the 4D functional image to the MNI template. Finally, high variance compounds were removed ${ }^{55}$ using nilearn ${ }^{56}$.

Creating an association matrix We then created an association matrix representing the strength of functional connectivity between pairs of brain regions. We defined regions of the SN, FPN, and DMN on a commonly applied parcellation scheme ${ }^{57}$. Coordinates of each region can be found in the supporting information. For each region, we extracted a time series of the BOLD signal separately for each individual. All regions were modeled as $10 \mathrm{~mm}$ diameter spheres around the center coordinates. The extracted time series were the average time series for all voxels within the sphere. We calculated the wavelet coherence matrix, $C$, using the extracted time series. Each element of $C_{i j}$ represented the magnitude squared coherence of the scale two $(0.0625-0.125 \mathrm{~Hz})$ Daubechies wavelet (length 4) decomposition of the time series of region $i$ and region $j$. We based our choice of frequency on previous work demonstrating sensitivity to neural processes $^{58-60}$.

Between-network connectivity We labeled regions by their putative functional systems. We calculated betweennetwork connectivity as the mean value of the association matrix elements representing functional connectivity between the three networks of interest. This procedure resulted in three between-system connectivity indices: DMN and SN connectivity, DMN and FPN connectivity, FPN and SN connectivity.

SN flexibility We next created a dynamic functional connectivity index that quantifies the extent to which nodes of the $\mathrm{SN}$ interact with nodes outside of its community (Fig. 2a-d). The time series for each brain region was divided into $T=15$ sliding time windows, each 20 TRs (30 s) in duration, with $50 \%$ overlap. The choice of window length was consistent with the majority of dynamic functional connectivity work to date employing 30-60 s windows, with most studies using 20 data points per window ${ }^{61}$. Within each window, edges between all nodes were estimated via wavelet coherence. The result was a time-ordered set of functional connectivity matrices for each subject.

We transformed the time-varying functional connectivity matrices into an ordered set of adjacency matrices, and subsequently into a multilayer network ${ }^{62,63}$. In this multilayer network, the graph in one time window is linked to the graph in adjacent time windows via identity edges that connect a node in one time window to the same node in neighboring time windows. We implemented multilayer modularity maximization in MATLAB ${ }^{64}$ and applied the procedure to each subject's functional connectivity matrices separately. The algorithm was applied with a default structural resolution parameter of 1 and an inter-layer strength parameter of 1 . As the algorithm is non-deterministic, we performed the optimization 100 times for each subject. This procedure resulted in $100 n \times m$ matrices for each participant, where $n$ is the number of nodes (264) and $m$ is the number of sliding windows (i.e., 15), indicating the community allegiance of each node during each sliding window. We used these matrices to create node flexibility indices. Node flexibility captures the number of times a node changes communities across time, normalized by the number of times the node could have changed communities $^{58}$. Using the Network Community Toolbox (http:// commdetect.weebly.com), node flexibility was calculated for each subject across each pipeline as the average flexibility value across the 100 iterations of the dynamic community detection procedure. SN flexibility was calculated as the average node flexibility over all nodes within the SN.

\section{Participant motion}

We calculated framewise displacement (FD) of the BOLD time series to provide an index of in-scanner motion during the sad mood induction ${ }^{65}$. We used mean FD for each participant as a covariate in analyses. We used the percent of volumes with excessive motion to test the robustness of the findings to the exclusion of participants with excessive motion (FD > 0.05; Supplementary Tables 2 and 3).

\section{Data analysis}

We tested the association among sadness and RNT, as well as the moderating effect of between-system 

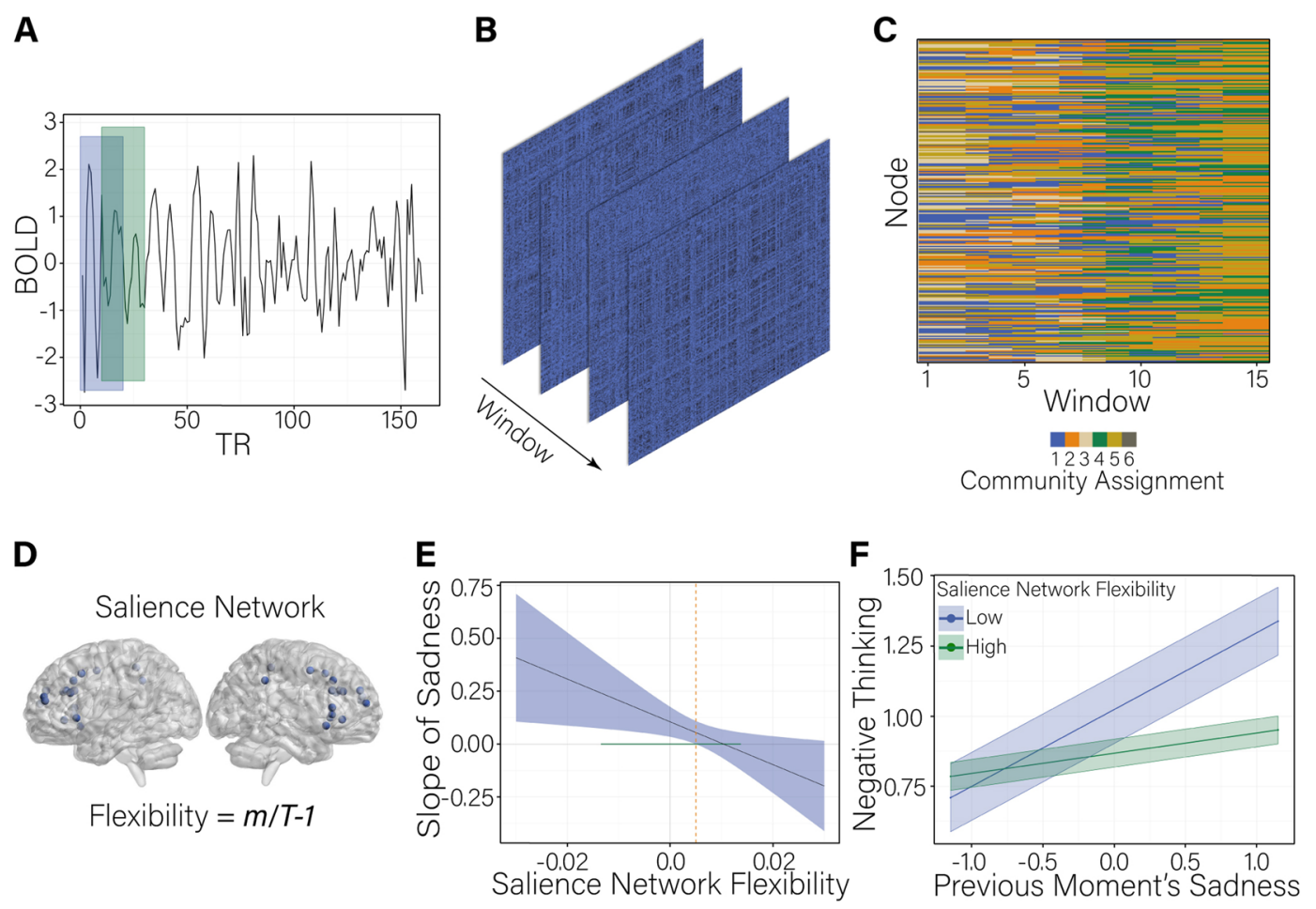

Fig. 2 Salience network flexibility construction and results. a Illustrates time courses of blood-oxygen-level-dependent (BOLD) signals from one of the 264 brain regions defined by ref. ${ }^{57}$. Functional connectivity between the time courses of each pair of brain regions is calculated via wavelet coherence within overlapping time windows of 20 TRs in length. b Illustrates connectivity matrices indicating the functional connectivity among each pair of brain regions. Four of the 15 sliding windows are illustrated. c Illustrates the construction of node flexibility. Multilayer modularity maximization is used to assign each brain region (node; $y$-axis) to a community (assignment indicated by color) at each sliding window ( $x$-axis). Panel $\mathbf{d}$ highlights the coordinates of regions in the salience network as defined by ref. ${ }^{57}$. Flexibility of an individual node captures the number of times the node changed community, normalized by the number of times the node could have changed communities. e lllustrates the values of salience network flexibility at which the association between previous moment's sadness and repetitive negative thinking is significant. The dashed orange vertical line at 0.005 indicates the value of salience network flexibility at which the effect of previous moment's sadness on negative thinking becomes non-significant. The upper bound of the region of significance for the salience network flexibility variable (0.04) is not shown as values below this bound are not observed in the sample. The range of values we observe in the sample is indicated by the horizontal green line at previous moment's sadness $=0$. The ribbon indicates the standard error of the mean. $\mathbf{f}$ Indicates the effect of previous moment's sadness on negative thinking at low and high levels of salience network flexibility. Low and high values of between-person, sample-mean centered salience network flexibility reflect plus and minus 1 standard deviation about the mean $(-0.03,0.03)$. Values for the previous moment's sadness on the $x$-axis reflect plus and minus 1 standard deviation about the mean. The slope of the simple regression of negative thinking on sadness at low levels of salience network flexibility is significant such that people with lower than average levels of salience network flexibility experienced significant increases in negative thinking following higher than usual levels of sadness at the previous measurement occasion. The slope of the simple regression at high levels of salience network flexibility is not significant. The ribbon indicates the standard error of the mean

functional connectivity and SN flexibility during sad mood induction, using a multilevel model to accommodate the nested nature of the data (i.e., repeated measures nested within persons) ${ }^{66}$. The sadness variable was lagged by one time point to create previous time point $(t-1)$ sadness scores. This process allowed us to capture the temporal precedence required to increase causal inference of the association between previous moment's sadness on current moment's RNT. The first measurement of each day was removed to ensure that the association between the previous moment's sadness and the current moment's RNT was approximately equally spaced. Measurement occasions with missing data for sadness and RNT were removed. Of 2030 possible total measurement assessments (58 participants * 35 occasions accounting for lagging sadness and removing the first measurement occasion of each day), 1995 (98.28\%) were available. The average time (in minutes) between assessments once the morning assessment was removed was $79.17 \mathrm{~min}(\mathrm{SD}=19.42)$. Results were robust to the inclusion of time points of ambulatory assessment data available per participant as a covariate (Supplementary Table 4) and no significant correlations emerged between completion rates and key study variables (all $p$-values $>0.05$ ).

We then separated the sadness variable into a withinperson and between-person variable to allow for both 
within-person and between-person inferences related to the association between sadness and $\mathrm{RNT}^{67}$. A timeinvariant, between-person sadness variable (SadnessBW) was calculated as the arithmetic mean across each individual's, grand-mean centered repeated measures. A timevarying, moment-level sadness variable (SadnessWN) was calculated as deviations from the between-person means. RNT was regressed on both the within-person and the between-person sadness variable, time in the study, DMN-SN, FPN-SN, DMN-FPN connectivity terms, SN flexibility, motion, sex, and depressive symptoms. Interactions among the within-person sadness variable and the functional connectivity terms were also included.

More specifically, we constructed the first level of the multilevel model as follows:

Level 1:

$$
\begin{gathered}
\text { Repetitive Negative Thinking } \\
+\beta_{1 i}=\beta_{0 i} \\
\text { Sadness } \mathrm{WN}_{i, t-1}+\beta_{2 i} \text { Time }_{i t}+e_{i t}
\end{gathered}
$$

where Repetitive Negative Thinking $i t$ is RNT for person $i$ at time $t, \beta_{0 i}$ indicates the average level of negative thinking for the prototypical individual in the sample, $\beta_{1 i}$ indicates within-person differences in RNT at time $t$ associated with sadness at time $t-1 ; \beta_{2 i}$ indicates the effect of time in the study on RNT; and $e_{i t}$ are timespecific residuals that were allowed to display autocorrelation (AR1).

Person-specific intercepts and associations from the Level 1 model were specified at the second level of the multilevel model, which we constructed as follows:

Level 2:

$$
\begin{gathered}
\beta_{0 i}=\gamma_{00}+\gamma_{01} \text { DefaultModeNetwork - SalienceNetwork }_{i} \\
+\gamma_{02} \text { DefaultModeNetwork }- \text { FrontoparietalNetwork }_{i} \\
+\gamma_{03} \text { FrontoparietalNetwork - SalienceNetwork }_{i} \\
+\gamma_{04} \text { SalienceNetworkFlexibility }_{i}+\gamma_{05} \text { Motion }_{i} \\
+\gamma_{06} \text { SadnessBW }_{i}+\gamma_{07} \text { Age }_{i}+\gamma_{08} \text { Sex }_{i}+\gamma_{09} \text { Depression }_{i}+u_{0 i} \\
\beta_{1 i}=\gamma_{10}+\gamma_{11}{\text { DefaultModeNetwork }- \text { SalienceNetwork }_{i}} \\
+\gamma_{12} \text { DefaultModeNetwork }- \text { FrontoparietalNetwork }_{i} \\
+\gamma_{13} \text { FrontoparietalNetwork - SalienceNetwork }_{i} \\
+\gamma_{14} \text { SalienceNetworkFlexibility }_{i}+u_{1 i} \\
\beta_{2 i}=\gamma_{20}+u_{2 i}
\end{gathered}
$$

where the $\gamma$ variables are sample-level parameters and the $u$ variables are residual between-person differences that may be correlated with one another but are uncorrelated with the variable $e_{i t}$. Parameters $\gamma_{01}$ to $\gamma_{09}$ indicate the effects of person-level DMN and SAL connectivity, DMN and FPN connectivity, FPN and SAL network connectivity, SAL flexibility, motion, usual sadness, age, sex, and depressive symptoms on RNT. Parameters $\gamma_{11}$ to $\gamma_{14}$ indicate how between-person differences in DMN and
SAL connectivity, DMN and FPN connectivity, FPN and SAL connectivity, and SAL flexibility moderated the association between the previous moment's sadness on the current moment's RNT.

We fit the model using lme4 in $\mathrm{R}^{68}$ with incomplete data being treated based on an assumption of being missing at random. We followed up on significant interactions using the Johnson-Neyman technique ${ }^{69,70}$ and plot simple slopes based on $+1 /-1$ standard deviation about the mean value of the moderator variable. Statistical significance was evaluated at an $\alpha=0.05$. Betweennetwork connectivity of the DMN and the $\mathrm{SN}$ was not a significant moderator (see Supplementary Table 5) and was removed from the final model.

\section{Results}

Table 1 provides descriptive statistics for the variables used in the analyses. Multilevel model results are presented in Table 2.

\section{FPN and SN connectivity moderates the association between sadness and RNT}

We observe an interaction between FPN and SN connectivity and the association between sadness and RNT $(\gamma=-11.15, p<0.001)$. The regression of RNT on sadness is significant and positive for participants with relatively weak connectivity among these systems (Fig. 3b; $\beta$ $=0.21$ (0.05), $p<0.001)$. Participants with low levels of FPN and SN connectivity experience increases in RNT following moments of higher than usual sadness (Fig. 3c). In contrast, participants with higher levels of FPN and SN connectivity show no moment-to-moment association between RNT and sadness $(\beta=0.01(0.05), p=0.89)$.

\section{DMN and FPN connectivity moderates the association between sadness and RNT}

We observe an interaction between DMN and FPN connectivity and the association between sadness and RNT $(\gamma=11.65, p=0.002)$. The regression of RNT on sadness is positive for participants with relatively high levels of connectivity between these networks (Fig. 3e; $\beta=0.22(0.02), p<0.001)$. Participants with high levels of DMN and FPN connectivity experience increases in RNT following moments of higher than usual sadness. In contrast, participants with low levels of DMN and FPN connectivity show no changes in RNT following moments of higher than usual sadness (Fig. 3f; $\beta=0.01(0.05)$, $p=0.83$ ).

\section{SN flexibility moderates the association between sadness and RNT}

We observe an interaction between SN flexibility and the association between sadness and RNT $(\gamma=-10.10$, $p=0.02$ ). The regression of RNT on previous moment's 
Table 1 Descriptive statistics and correlations among variables used in the multilevel model

\begin{tabular}{|c|c|c|c|c|c|c|c|c|c|}
\hline Variables & 1 & 2 & 3 & 4 & 5 & 6 & 7 & 8 & 9 \\
\hline 1. Sadness (AA) & 1 & & & & & & & & \\
\hline 2. Negative thinking (AA) & $0.84^{* * *}$ & 1 & & & & & & & \\
\hline 3. Depressive symptoms & $0.55^{* * *}$ & $0.61^{* * *}$ & 1 & & & & & & \\
\hline 4. DMN-FPN & -0.17 & -0.21 & -0.18 & 1 & & & & & \\
\hline 5. DMN-SAL & -0.07 & -0.18 & $-0.27^{*}$ & $0.45^{* * *}$ & 1 & & & & \\
\hline 6. FPN-SAL & -0.09 & -0.15 & -0.18 & $0.48^{* * *}$ & $0.51^{* * *}$ & 1 & & & \\
\hline 7. SAL flexibility & -0.06 & 0.007 & 0.17 & $-0.27^{*}$ & $-0.32^{*}$ & $-0.43^{* * *}$ & 1 & & \\
\hline 8. Age & 0.04 & 0.07 & -0.07 & 0.12 & 0.01 & 0.15 & -0.04 & 1 & \\
\hline 9. Motion & -0.001 & 0.01 & 0.13 & -0.003 & -0.18 & 0.004 & 0.13 & $0.54^{* * *}$ & 1 \\
\hline Variables & 1 & 2 & 3 & 4 & 5 & 6 & 7 & 8 & 9 \\
\hline Mean & 0.00 & 0.91 & -0.02 & 0.18 & 0.18 & 0.19 & 0.23 & 36.69 & 0.25 \\
\hline Standard deviation & 1.07 & 1.02 & 0.95 & 0.01 & 0.01 & 0.01 & 0.01 & 10.66 & 0.11 \\
\hline
\end{tabular}

$N=58$

$A A$ Ambulatory assessment, Negative thinking the person-level average of repetitive negative thinking across all measurement occasions, Sadness between-person version of the sadness variable used in the multilevel model, DMN default mode network, FPN fronto-parietal network, SAL salience network

${ }^{* * *} p<0.001 ;{ }^{* *} p<0.01 ;{ }^{*} p<0.05$

sadness is positive for participants with low values of SN flexibility (Fig. 2e; $\beta=0.21$ (0.07), $p=0.004$ ). Participants with low levels of SN flexibility experience increases in RNT following moments of higher than usual sadness (Fig. 2f). In contrast, participants with higher levels of SN flexibility show no moment-to-moment association between RNT and sadness $(\beta=0.04$ (0.03), $p=0.90)$.

\section{Additional analyses}

Additional analyses confirm that results are robust to (i) the removal of non-significant covariates (Supplementary Table 6), (ii) the inclusion of group status (remitted depressed versus healthy controls) rather than the continuous measure of depressive symptoms (Supplementary Table 7), (iii) the inclusion of previous moment's RNT to account for any covariation between RNT and sadness at time $t$-1 (i.e., establishing causality in the sense of Granger; Supplementary Table 8), and (iv) including interactions with depression group indicating that results hold irrespective of group status, although we note that the sample may be underpowered to detect significant threeway interactions (Supplementary Table 9). In all cases, the moment-to-moment association between sadness and RNT is moderated by connectivity among the DMN and FPN, connectivity among SAL and FPN, and SAL flexibility in a manner consistent with the main analyses.

\section{Discussion}

RNT is a maladaptive response to sadness. Characterizing between-person differences associated with RNT tendencies will facilitate attempts to promote more adaptive responses to sadness. Building on the impaired disengagement hypothesis ${ }^{12}$ and empirical support for the role of cognitive flexibility in moderating the tendency to engage in $\mathrm{RNT}^{14}$, we tested the moderating role of functional connectivity among brain networks associated with cognitive control ${ }^{15}$. We show that functional connectivity among the DMN, FPN, and SN during sad mood moderates the moment-to-moment association between sadness and RNT in daily life. Increased between-network connectivity of the DMN and the FPN, and decreased between-network connectivity of the SN and the FPN, are associated with increases in RNT following increases in sadness. We also observe that participants with flexible engagement of the SN show no tendency to increase RNT following increases in sadness. Between-network connectivity of the $\mathrm{SN}$ and DMN does not moderate the association between sadness and RNT. The findings are consistent with the impaired disengagement hypothesis and highlight potential roles for cognitive conflict signaling and attentional control in inhibiting $\mathrm{RNT}^{13,14}$. Individuals with greater ability to identify cognitive conflict and to engage executive functions may be better able to inhibit perseverative thinking and to divert attention away from their negative mood to a greater extent than individuals with impairments in these functions.

The pattern of findings for DMN and FPN connectivity, and for FPN and SN connectivity, is in line with previous work indicating that greater strength of connectivity between the DMN and FPN is associated with poorer 
Table 2 Results of the multilevel model examining associations among sadness and repetitive regative thinking, and its moderation by between-network functional connectivity and salience network flexibility

\begin{tabular}{|c|c|c|c|c|c|}
\hline \multicolumn{6}{|l|}{ Fixed effects } \\
\hline & \multicolumn{2}{|l|}{ Estimate } & \multicolumn{2}{|l|}{ Standard error } & $p$-value \\
\hline Intercept & \multicolumn{2}{|l|}{$1.13^{* * *}$} & \multicolumn{2}{|l|}{0.16} & $<0.001$ \\
\hline Sadness WN & \multicolumn{2}{|l|}{$0.11^{* *}$} & \multicolumn{2}{|l|}{0.04} & 0.004 \\
\hline Time & \multicolumn{2}{|l|}{-0.001} & \multicolumn{2}{|l|}{0.004} & 0.80 \\
\hline DMN-FPN & \multicolumn{2}{|l|}{-0.77} & \multicolumn{2}{|l|}{11.04} & 0.94 \\
\hline FPN-SAL & \multicolumn{2}{|l|}{-7.66} & \multicolumn{2}{|l|}{7.75} & 0.33 \\
\hline SAL flexibility & \multicolumn{2}{|l|}{-7.80} & \multicolumn{2}{|l|}{13.20} & 0.56 \\
\hline Motion & \multicolumn{2}{|l|}{-0.02} & \multicolumn{2}{|l|}{0.72} & 0.98 \\
\hline Sadness BW & \multicolumn{2}{|l|}{$0.68^{* * *}$} & \multicolumn{2}{|l|}{0.08} & $<0.001$ \\
\hline Age & \multicolumn{2}{|l|}{0.01} & \multicolumn{2}{|l|}{0.01} & 0.37 \\
\hline Sex & \multicolumn{2}{|l|}{-0.29} & \multicolumn{2}{|l|}{0.15} & 0.05 \\
\hline Depressive symptoms & \multicolumn{2}{|l|}{$0.19^{*}$} & \multicolumn{2}{|l|}{0.09} & 0.03 \\
\hline DMN-FPN $\times$ Sadness WN & \multicolumn{2}{|l|}{$11.65^{* *}$} & \multicolumn{2}{|l|}{3.81} & 0.002 \\
\hline FPN-SAL $\times$ Sadness WN & \multicolumn{2}{|l|}{$-11.15^{* * *}$} & \multicolumn{2}{|l|}{2.84} & $<0.001$ \\
\hline SAL Flexibility $\times$ Sadness WN & \multicolumn{2}{|l|}{$-10.10^{*}$} & \multicolumn{2}{|l|}{4.28} & 0.02 \\
\hline \multicolumn{6}{|l|}{ Random effects } \\
\hline & \multicolumn{3}{|c|}{ Estimate } & Confidence interval & \\
\hline Intercept & & 0.48 & & $0.38-0.61$ & \\
\hline Sadness WN & & 0.06 & & $0.01-0.27$ & \\
\hline Time & & 0.03 & & $0.02-0.3$ & \\
\hline Correlation (Intercept, Sadness WN) & & 0.20 & & $-0.56-0.78$ & \\
\hline Correlation (Intercept, Time) & & 0.41 & & $0.03-0.69$ & \\
\hline Correlation (Sadness WN, Time) & & 0.10 & & $-0.43-0.57$ & \\
\hline AR1 & & 0.03 & & $-0.02-0.08$ & \\
\hline Residual & & 1.09 & & $1.06-1.3$ & \\
\hline
\end{tabular}

Fit indices

\begin{tabular}{ll}
\hline AIC & 6250.58 \\
BIC & 6373.59 \\
\hline
\end{tabular}

Continuous predictors were sample-mean centered and time was centered at the middle of the ambulatory assessment protocol to facilitate interpretation of the intercept. Sex was specified as a factor with $1=$ male, $2=$ female; the $N=1995$ observations were nested within 58 participants

Sadness WN within-person deviated version of sadness, Sadness BW between-person version of sadness, Depressive symptoms composite score by averaging zstandardized BDI-II and MADRS scores, DMN default mode network, FPN fronto-parietal network, SAL salience network, AR1 autocorrelation, AIC Akaike information criterion, BIC Bayesian information criterion

${ }^{* * *} p<0.001,{ }^{* *} p<0.01,{ }^{*} p<0.05$

cognitive control performance ${ }^{23}$ and that the SN plays a causal role in switching between FPN and DMN activity ${ }^{27}$. The specificity of the association for FPN and SN network connectivity and not DMN and SN connectivity, however, indicates more complex interactions among the three systems than is currently appreciated in the broader literature. Recent work provides insight into these complex interactions ${ }^{71}$. During an $n$-back working memory task, 
A

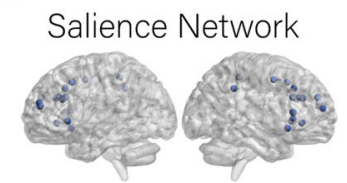

Frontoparietal Network

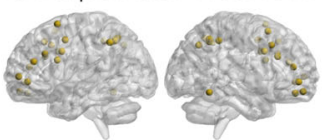

D

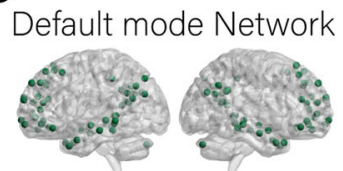

Frontoparietal Network

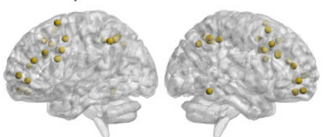

B

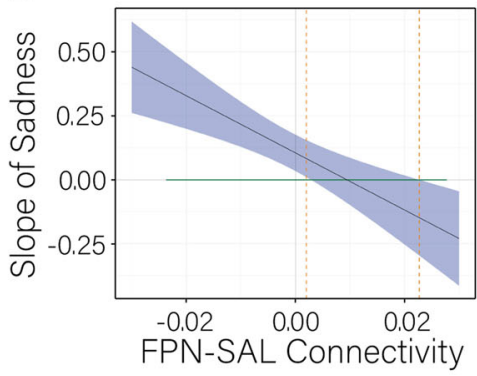

E

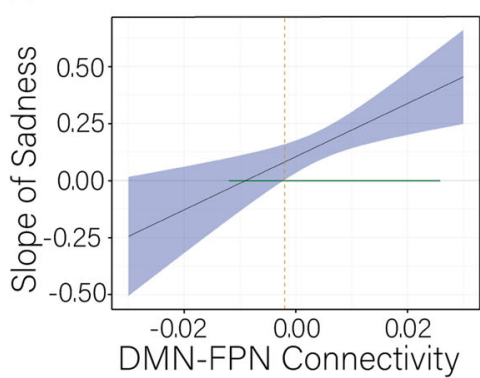

C

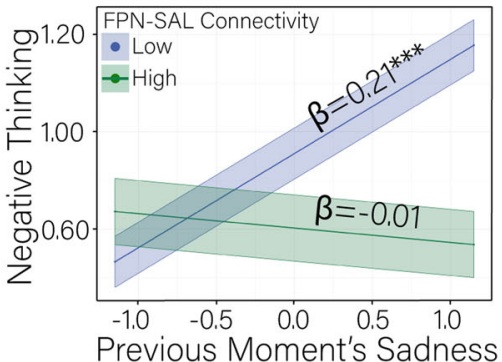

$\mathbf{F}$

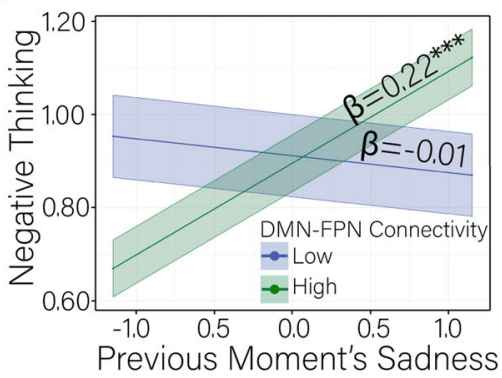

Fig. 3 The conditional relation between sadness and repetitive negative thinking as a function of between-person differences in default mode, fronto-parietal, and salience network connectivity. a Highlights the coordinates of regions in the salience network and the fronto-parietal network as defined by ref. ${ }^{57}$. $\mathbf{b}$ Illustrates the values of fronto-parietal network and salience network connectivity (FPN-SAL) at which the association between previous moment's sadness and repetitive negative thinking is significant. The dashed orange vertical line at 0.003 indicates the value of FPN-SAL connectivity at which the effect of previous moment's sadness on negative thinking becomes non-significant. The upper bound of the region of significance for the FPN-SAL variable (0.02) is also shown. The range of values we observe in the sample is indicated by the horizontal green line at slope of sadness $=0$. The ribbon indicates the standard error of the mean. $\mathbf{c}$ Indicates the effect of previous moment's sadness on negative thinking at low and high levels of FPN-SAL connectivity. Low and high values of between-person, sample-mean centered FPN-SAL connectivity reflect plus and minus 1 standard deviation about the mean $(-0.01,0.01)$. Values for the previous moment's sadness on the $x$-axis reflect plus and minus 1 standard deviation about the mean. The slope of the simple regression of negative thinking on sadness at low levels of FPN-SAL connectivity is significant such that people with lower than average levels of FPN-SAL connectivity experienced significant increases in negative thinking following higher than usual levels of sadness. The slope of the simple regression at high levels of FPN-SAL connectivity is not significant. The ribbon indicates the standard error of the mean. $\mathbf{d}$ Highlights the coordinates of regions in the default mode network and the fronto-parietal network as defined by ref. ${ }^{57}$. e lllustrates the values of default mode network and fronto-parietal network connectivity (DMN-FPN) at which the association between previous moment's sadness and negative thinking is significant. The dashed orange vertical line at -0.002 indicates the lower bound of the region of significance on the DMN-FPN connectivity variable at which point the effect of previous moment's sadness on negative thinking becomes significant. The upper bound is beyond the range of observed data and is not shown. The range of values observed in the sample is indicated by the horizontal green line at sadness $=0$. The ribbon indicates the standard error of the mean. $\mathbf{f}$ Indicates the association between previous moment's sadness and negative thinking at low and high levels of DMN-FPN connectivity. Low and high values of between-person, sample-mean centered DMN-FPN connectivity are defined as plus and minus 1 standard deviation about the mean $(-0.01,0.01)$. Values for the previous moment's sadness on the $x$-axis reflect plus and minus 1 standard deviation about the mean. The slope of the simple regression at high levels of DMN-FPN connectivity is significant such that people with higher than average levels of DMN-FPN connectivity experienced significant increases in negative thinking following higher than usual levels of sadness at the previous measurement occasion. The slope of the simple regression of negative thinking on sadness is not significant at low levels of DMN-FPN connectivity. The ribbon indicates the standard error of the mean

increased connectivity between the FPN and DMN was associated with poorer performance. Coupling both levels of activation and connectivity within a dynamical model designed to probe the association between system activity and connectivity, there was no evidence that activity of the DMN was associated with the functional coupling between the DMN and FPN. In contrast, modulating FPN amplitude impacted the strength of connectivity between DMN and FPN. These findings suggest that connectivity between the SAL and FPN may be particularly important for cognitive control, with changes in activity in the FPN in response to signals from the SAL leading to changes in connectivity between the FPN and DMN and, in turn, changes in cognitive control abilities.

A notable finding is that SN flexibility was protective against experiencing increases in RNT following increases in sadness. This finding provides further support for a putative role of cognitive flexibility in understanding maladaptive responses to depressed mood. SN flexibility captures the extent to which nodes of the $\mathrm{SN}$ interact with 
nodes in other communities. Nodes that change communities many times may moderate multiple processes and may be essential for dynamic and adaptive processes ${ }^{58}$. Indeed, flexibility of the $\mathrm{SN}$ is associated with cognitive flexibility $^{34}$. We find that $\mathrm{SN}$ inflexibility is associated with increases in RNT following sadness above and beyond time-invariant (i.e., static) functional connectivity indices, in line with the increasing recognition of the time-varying nature of brain network organization ${ }^{72}$ and its importance for understanding human mood and cognition ${ }^{59,73-77}$.

\section{Limitations}

It is important to consider the findings in light of the study's strengths and limitations. Our measure of RNT captures perceived uncontrollability over negative thoughts. Worry and rumination are two forms of RNT that have received extensive treatment in the literature, with worry being more future-focused and rumination more pastfocused $^{78}$. Examining the content of RNT will allow insight into the specificity of our findings to worry and rumination, though it has been argued that common processes exist across different forms of $\mathrm{RNT}^{79,80}$. Connectivity among the DMN, FPN, and SN provides a general index of cognitive flexibility but specific cognitive functions were not examined. Working memory has emerged as a particularly important function for understanding RNT and an examination of its neural correlates in relation to RNT during the course of daily life will be an important avenue for future research. The extent to which patterns of functional connectivity during sad mood induction specifically drives the results will require further investigation, especially since emotional states modulate cognitive flexibility ${ }^{81}$, including in the context of remitted depression ${ }^{38}$.

\section{Conclusions}

Functional connectivity among the DMN, the FPN, and the $\mathrm{SN}$, as well as $\mathrm{SN}$ flexibility, during sad mood induction moderates the lagged, moment-to-moment association between sadness and RNT in daily life. The findings highlight the utility of large-scale functional brain networks with roles in cognitive conflict signaling, selfreferential thought, and cognitive flexibility above and beyond self-report and interviewer-rated depressive symptoms in understanding the cognitive-affective sequelae of sadness. Results also indicate the feasibility and importance of considering moment-to-moment interactions between affect and cognition in daily life and encourages their consideration in cognitive control training interventions ${ }^{82-84}$ as well as in mindfulness-based interventions ${ }^{85}$ designed to reduce RNT.

\section{Acknowledgements}

C.K. and P.K. would like to acknowledge support from the Deutsche Forschungsgemeinschaft (DFG) through grants KU1464/4-1,2 and PK576/121,2. D.S.B. and D.M.L. would like to acknowledge support from the John D. and
Catherine T. MacArthur Foundation, the Alfred P. Sloan Foundation, the Paul G. Allen Foundation, the Army Research Laboratory through contract number W911NF-10-2-0022, the Army Research Office through contract numbers W91 1NF-14-1-0679 and W911NF-16-1-0474, the National Institute of Health (2R01-DC-009209-11, 1R01HD086888-01, R01-MH107235, R01-MH107703, R01MH109520, 1R01NS099348, and R21-M MH-106799), the Office of Naval Research, and the National Science Foundation (BCS-1441502, CAREER PHY1554488, BCS-1631550, and CNS-1626008). The content is solely the responsibility of the authors and does not necessarily represent the official views of any of the funding agencies.

\section{Author details}

'Department of Bioengineering, School of Engineering and Applied Sciences, University of Pennsylvania, Philadelphia, PA 19104, USA. ${ }^{2}$ Research Group Longitudinal and Intervention Research, Department of Psychiatry and Psychotherapy, Central Institute of Mental Health, Medical Faculty Mannheim, Heidelberg University, 68159 Mannheim, Germany. ${ }^{3}$ Department of Clinical Psychology, Central Institute of Mental Health, Medical Faculty Mannheim, Heidelberg University, 68159 Mannheim, Germany. ${ }^{4}$ Institute Psychiatric and Psychosomatics Psychotherapy, Central Institute of Mental Health, Medical Faculty Mannheim, Heidelberg University, 68159 Mannheim, Germany. ${ }^{5}$ Department of Electrical \& Systems Engineering, School of Engineering and Applied Sciences, University of Pennsylvania, Philadelphia, PA 19104, USA.

${ }^{6}$ Department of Neurology, Perelman School of Medicine, University of Pennsylvania, Philadelphia, PA 19104, USA. 'Department of Physics \& Astronomy, College of Arts and Sciences, University of Pennsylvania, Philadelphia, PA 19104, USA. ${ }^{8}$ Department of Psychiatry, Perelman School of Medicine, University of Pennsylvani, Philadelphia, PA 19104, USA. 'Santa Fe Institute, Santa Fe, NM 87501, USA

\section{Code availability}

Code used throughout for data processing and analysis are available from the corresponding author

\section{Conflict of interest}

The authors declare that they have no conflict of interest.

\section{Publisher's note}

Springer Nature remains neutral with regard to jurisdictional claims in published maps and institutional affiliations.

Supplementary Information accompanies this paper at (https://doi.org/ 10.1038/s41398-019-0560-0).

Received: 4 October 2018 Revised: 11 April 2019 Accepted: 13 May 2019 Published online: 18 September 2019

\section{References}

1. Carver, C. S. \& Scheier, M. F. On the Self-regulation of Behavior. (Cambridge University Press, New York, NY, 1998).

2. Nolen-Hoeksema, S. Responses to depression and their effects on the duration of depressive episodes. J. Abnorm Psychol. 100, 569-582 (1991).

3. Nolen-Hoeksema, S., Wisco, B. E. \& Lyubomirsky, S. Rethinking rumination. Perspect. Psychol. Sci. 3, 400-424 (2008).

4. Ruscio, A. M., Seitchik, A. E., Gentes, E. L., Jones, J. D. \& Hallion, L. S. Perseverative thought: a robust predictor of response to emotional challenge in generalized anxiety disorder and major depressive disorder. Behav. Res. Ther. 49, 867-874 (2011).

5. Just, N. \& Alloy, L. B. The response styles theory of depression: tests and an extension of the theory. J. Abnorm. Psychol. 106, 221-229 (1997).

6. Slavish, D. C. \& Graham-Engeland, J. E. Rumination mediates the relationships between depressed mood and both sleep quality and self-reported health in young adults. J. Behav. Med. 38, 204-213 (2015).

7. Kuehner, C. Why is depression more common among women than among men? Lancet Psychiatry 4, 146-158 (2017).

8. Lyubomirsky, S., Layous, K., Chancellor, J. \& Nelson, S. K. Thinking about rumination: the scholarly contributions and intellectual legacy of Susan NolenHoeksema. Annu. Rev. Clin. Psychol. 11, 1-22 (2015). 
9. Nolen-Hoeksema, S. \& Watkins, E. A heuristic for developing transdiagnostic models of psychopathology: explaining multifinality and divergent trajectories. Perspect. Psychol. Sci. 6, 589-609 (2011).

10. Ehring, T. \& Watkins, E. R. Repetitive negative thinking as a transdiagnostic process. Int J. Cogn. Ther. 1, 192-205 (2008).

11. McEvoy, P. M., Watson, H., Watkins, E. R. \& Nathan, P. The relationship between worry, rumination, and comorbidity: evidence for repetitive negative thinking as a transdiagnostic construct. J. Affect Dis. 151, 313-320 (2013).

12. Koster, E. H., De Lissnyder, E., Derakshan, N. \& De Raedt, R. Understanding depressive rumination from a cognitive science perspective: the impaired disengagement hypothesis. Clin. Psychol. Rev. 31, 138-145 (2011).

13. Whitmer, A. J. \& Banich, M. T. Inhibition versus switching deficits in different forms of rumination. Psychol. Sci. 18, 546-553 (2007).

14. De Lissnyder, E. et al. Cognitive control moderates the association between stress and rumination. J. Behav. Ther. Exp. Psychiatry 43, 519-525 (2012).

15. Bonnelle, $V$. et al. Salience network integrity predicts default mode network function after traumatic brain injury. Proc. Natl Acad. Sci. USA 109, 4690-4695 (2012).

16. Mazoyer, B. et al. Cortical networks for working memory and executive functions sustain the conscious resting state in man. Brain Res. Bull. 54, 287-298 (2001)

17. Shulman, G. L. et al. Common blood flow changes across visual tasks: II. Decreases in cerebral cortex. J. Cog. Neurosci. 9, 648-663 (1997).

18. Curtis C. E. \& D'Esposito M. The inhibition of unwanted actions. In J. Bargh, P. Gollwitzer, \& E. Moresella (Eds.), The Psychology of Action (pp.72-97). (Guilford Press, New York, NY, 2008).

19. Mars, R. B. \& Grol, M. J. Dorsolateral prefrontal cortex, working memory, and prospective coding for action. J. Neurosci. 27, 1801-1802 (2007).

20. Shomstein, S., Kravitz, D. J. \& Behrmann, M. Attentional control: temporal relationships within the fronto-parietal network. Neuropsychologia $\mathbf{5 0}$ 1202-1210 (2012).

21. Jiang, Y. \& Kanwisher, N. Common neural substrates for response selection across modalities and mapping paradigms. J. Cog. Neurosci. 15, 1080-1094 (2003).

22. Ridderinkhof, K. R., Van Den Wildenberg, W. P., Segalowitz, S. J. \& Carter, C. S. Neurocognitive mechanisms of cognitive control: the role of prefrontal cortex in action selection, response inhibition, performance monitoring, and rewardbased learning. Brain Cogn. 56, 129-140 (2004).

23. Hampson, M., Driesen, N., Roth, J. K., Gore, J. C. \& Constable, R. T. Functional connectivity between task-positive and task-negative brain areas and its relation to working memory performance. Magn. Res. Imag. 28, 1051-1057 (2010).

24. Kelly, A. C., Uddin, L. Q., Biswal, B. B., Castellanos, F. X. \& Milham, M. P. Competition between functional brain networks mediates behavioral variability. Neuroimage 39, 527-537 (2008).

25. Uddin, L. Q. Salience processing and insular cortical function and dysfunction. Nat. Rev. Neurosci. 16, 55-61 (2015).

26. Menon, V. Salience network. Brain Mapp.: Encyclopedic Ref. 2, 597-611 (2015).

27. Sridharan, D., Levitin, D. J. \& Menon, V. A critical role for the right fronto-insular cortex in switching between central-executive and default-mode networks. Proc. Natl Acad. Sci. USA 105, 12569-12574 (2008).

28. Broyd, S. J. et al. Default-mode brain dysfunction in mental disorders: a systematic review. Neurosci. Biobehav. Rev. 33, 279-296 (2009).

29. Whitfield-Gabrieli, S. \& Ford, J. M. Default mode network activity and connectivity in psychopathology. Annu. Rev. Clin. Psychol. 8, 49-76 (2012).

30. Lydon-Staley D. M. \& Bassett D. S. in Current Topis in Behavioral Neuroscienes: Biomarkers in Psychiatry (eds Pratt, J. \& Halls, J) (Springer, Berlin, Germany, 2018)

31. Hamilton, J. P. et al. Default-mode and task-positive network activity in major depressive disorder: implications for adaptive and maladaptive rumination. Biol. Psychiatry 70, 327-333 (2011).

32. Burrows, C. A., Timpano, K. R. \& Uddin, L. Q. Putative brain networks underlying repetitive negative thinking and comorbid internalizing problems in autism. Clin. Psychol. Sci. 5, 522-536 (2017).

33. Koster, E. H. et al. Examining the relation between mood and rumination in remitted depressed individuals: a dynamic systems analysis. Clin. Psychol. Sci. 3 , 619-627 (2015).

34. Chen, T., Cai, W., Ryali, S., Supekar, K. \& Menon, V. Distinct global brain dynamics and spatiotemporal organization of the salience network. PLoS Biol. 14, e1002469 (2016)
35. Nomi, J. S. et al. Dynamic functional network connectivity reveals unique and overlapping profiles of insula subdivisions. Hum. Brain Mapp. 37, 1770-1787 (2016).

36. Huffziger, S. et al. Effects of mood and rumination on cortisol levels in daily life: An ambulatory assessment study in remitted depressed patients and healthy controls. Psychoneuroendocrinology 38, 2258-2267 (2013).

37. Timm, C. et al. Cognitive and affective trait and state factors influencing the long-term symptom course in remitted depressed patients. PlOS ONE 12, e0178759 (2017).

38. Zamoscik, V., Huffziger, S., Ebner-Priemer, U., Kuehner, C. \& Kirsch, P. Increased involvement of the parahippocampal gyri in a sad mood predicts future depressive symptoms. Soc. Cogn. Affect Neurosci. 9, 2034-2040 (2014).

39. Zamoscik, V. E. et al. Respiration pattern variability and related default mode network connectivity are altered in remitted depression. Psychol. Med. 48, 2364-2374 (2018)

40. Wittchen, H. U., Wunderlich, U., Gruschwitz, S. \& Zaudig, M. SCID: Structured Clinical Interview for DSM-IV Axis I Disorders. (Hogrefe, Göttingen, 1997).

41. Trull, T. J. \& Ebner-Priemer, U. Ambulatory Assessment. Annu Rev. Clin. Psychol. 9. 151-176 (2013).

42. Raes, F., Hermans, D., Williams, J. M. G., Bijttebier, P. \& Eelen, P. A "Triple W"model of rumination on sadness: why am I feeling sad, what's the meaning of my sadness, and wish I could stop thinking about my sadness (but I can't!). Cogn. Ther. Res. 32, 526-541 (2008)

43. Kuehner, C., Welz, A., Reinhard, I. \& Alpers, G. W. Lab meets real life: A laboratory assessment of spontaneous thought and its ecological validity. PloS ONE 12, e0184488 (2017).

44. Beck, A. T., Steer, R. A. \& Brown, G. K. Manual for the Beck Depression Inventory-ll. (The Psychological Corporation, San Antonio, 1996).

45. Hautzinger, M., Keller, F., Kuehner, C. \& Beck, A. T. Das Beck Depressionsinventar II. Deutsche Bearbeitung und Handbuch zum BDI II. (Harcout Test Services, Frankfurt a.M., 2006).

46. Montgomery, S. A. \& Asberg, M. A new depression scale designed to be sensitive to change. Br. J. Psychiatry 134, 382-389 (1979).

47. Schmidtke, A., Fleckenstein, P., Moises, W. \& Beckmann, H. Studies of the reliability and validity of the German version of the Montgomery-Asberg Depression Rating Scale (MADRS). Schweiz Arch. Neurol. Psychiatr. 139, 51-65 (1988).

48. Kühner, C., Bürger, C., Keller, F. \& Hautzinger, M. Reliabilität und Validität des revidierten Beck-Depressionsinventars (BDI-II). Der Nervenarzt 78, 651-656 (2007).

49. Huffziger, S., Reinhard, I. \& Kuehner, C. A longitudinal study of rumination and distraction in formerly depressed inpatients and community controls. J. Abnorm. Psychol. 118, 746-756 (2009).

50. Ciric, R. et al. Benchmarking of participant-level confound regression strategies for the control of motion artifact in studies of functional connectivity. Neurolmage 154, 174-187 (2017).

51. Lydon-Staley, D. M., Ciric, R., Satterthwaite, T. D. \& Bassett, D. S. Evaluation of confound regression strategies for the mitigation of motion artifact in studies of dynamic resting state functional connectivity. Netw. Neurosci. 3, 427-454 (2019).

52. Cox, R. W. AFNl: software for analysis and visualization of functional magnetic resonance neuroimages. Comput. Biomed. Res. 29, 162-173 (1996).

53. Jenkinson, M., Beckmann, C. F., Behrens, T. E., Woolrich, M. W. \& Smith, S. M. Fsl. Neurolmage 62, 782-790 (2012).

54. Avants, B. B., Tustison, N. \& Song, G. Advanced normalization tools (ANTS) Insight J. 2, 1-35 (2009).

55. Behzadi, Y., Restom, K., Liau, J. \& Liu, T. T. A component based noise correction method (CompCor) for BOLD and perfusion based fMRI. Neurolmage 37, 90-101 (2007).

56. Abraham, A. et al. Machine learning for neuroimaging with scikit-learn. Front Neuroinform. 8, 14 (2014).

57. Power, J. D. et al. Functional network organization of the human brain. Neuron 72, 665-678 (2011).

58. Bassett, D. S. et al. Dynamic reconfiguration of human brain networks during learning. Proc. Natl Acad. Sci. USA 108, 7641-7646 (2011).

59. Betzel R. F., Satterthwaite T. D., Gold J. I. \& Bassett D. S. Positive affect, suprise, and fatigue are correlates of network flexibility. Sci. Rep. 7, 520 (2017).

60. Zhang, Z., Telesford, Q. K., Giusti, C., Lim, K. O. \& Bassett, D. S. Choosing wavelet methods, filters, and lengths for functional brain network construction. PloS ONE 11, e0157243 (2016). 
61. Preti, M. G., Bolton, T. A. W. \& Van De Ville, D. The dynamic functional connectome: State-of-the-art and perspectives. Neurolmage 160, 41-54 (2017).

62. Bassett, D. S. et al. Robust detection of dynamic community structure in networks. Chaos 23, 013142 (2013)

63. Mucha, P. J., Richardson, T., Macon, K., Porter, M. A. \& Onnela, J. P. Community structure in time-dependent, multiscale, and multiplex networks. Science $\mathbf{3 2 8}$ 876-878 (2010).

64. Jeub L. G. S., Bazzi M., Jutla I. S. \& Mucha P. J. A generalized Louvain method for community detection implemented in MATLAB. http://netwiki.amath.unc. edu/GenLouvain/GenLouvain (2011).

65. Power, J. D., Barnes, K. A., Snyder, A. Z., Schlaggar, B. L. \& Petersen, S. E. Spurious but systematic correlations in functional connectivity MRI networks arise from subject motion. Neurolmage 59, 2142-2154 (2012).

66. Snijders, T. A. B. \& Bosker, R. J. Multilevel Analysis: an Introduction to Basic and Advanced Multilevel Modeling. 2nd edn. (Sage Publishers, London, UK, 2012).

67. Bolger, N. \& Laurenceau, J.P. Intensive Longitudinal Methods: an Introduction to Diary and Experience Sampling Research. (Guilford Press, New York, NY, 2013).

68. Bates, D., Maechler, M., Bolker, B. \& Walker, S. Fitting Linear Mixed-Effects Models Using Ime4. J. Stat. Softw. 67, 1-48 (2015).

69. Bauer, D. J. \& Curran, P. J. Probing interactions in fixed and multilevel regression: inferential and graphical techniques. Multivar. Behav. Res. 40, 373-400 (2005).

70. Johnson, P. O. \& Neyman, J. Tests of certain linear hypotheses and their applications to some educational problems. Stat. Res. Mem. 1, 57-93 (1936).

71. Murphy A. C., Bertolero M. A., Papadopoulos L., Lydon-Staley D. M. \& Bassett D. S. Multiscale and multimodal network dynamics underpinning working memory. Preprint at https:/arxiv.org/abs/1901.06552 (2019).

72. Calhoun, V. D., Miller, R., Pearlson, G. \& Adalı, T. The chronnectome: timevarying connectivity networks as the next frontier in fMRI data discovery. Neuron 84, 262-274 (2014).

73. Braun, $U$. et al. Dynamic reconfiguration of frontal brain networks during executive cognition in humans. Proc. Natl Acad. Sci. USA 112, 11678-11683 (2015).
74. Chai, L. R., Mattar, M. G., Blank, I. A., Fedorenko, E. \& Bassett, D. S. Functional network dynamics of the language system. Cereb. Cortex 26, 4148-4159 (2016).

75. Gerraty, R. T. et al. Dynamic flexibility in striatal-cortical circuits supports reinforcement learning. J. Neurosci. 38, 2442-2453 (2018).

76. He, $X$. et al. Disrupted dynamic network reconfiguration of the language system in temporal lobe epilepsy. Brain 141, 1375-1389 (2018).

77. Shine, J. M., Koyejo, O. \& Poldrack, R. A. Temporal metastates are associated with differential patterns of time-resolved connectivity, network topology, and attention. Proc. Natl Acad. Sci. USA 113, 9888-9891 (2016).

78. Watkins, E. R. Constructive and unconstructive repetitive thought. Psychol. Bull. 134, 163-206 (2008).

79. McEvoy, P. M., Mahoney, A. E. \& Moulds, M. L. Are worry, rumination, and postevent processing one and the same? Development of the Repetitive Thinking Questionnaire. J. Anxiety Disord. 24, 509-519 (2010).

80. Harvey, A. G., Watkins, E., Mansell, W. \& Shafran, R. Cognitive Behavioural Processes Across Psychological Disorders: A Transdiagnostic Approach to Research and Treatment. (Oxford University Press, Oxford, UK, 2004).

81. Mueller, S. The influence of emotion on cognitive control: relevance for development and adolescent psychopathology. Front. Psychol. 2, 1-21 (2011).

82. Siegle, G. J. et al. You gotta work at it: pupillary indices of task focus are prognostic for response to a neurocognitive intervention for rumination in depression. Clin. Psychol. Sci. 2, 455-471 (2014).

83. Motter, J. N. et al. Computerized cognitive training and functional recovery in major depressive disorder: a meta-analysis. J. Affect. Disord. 189, 184-19 (2016).

84. Hoorelbeke, K., Koster, E. H., Vanderhasselt, M. A., Callewaert, S. \& Demeyer, The influence of cognitive control training on stress reactivity and rumination in response to a lab stressor and naturalistic stress. Behav. Res. Ther. 69, 1-10 (2015).

85. Timm, C. et al. Mindfulness-Based Attention Training improves Cognitive and Affective Processes in Daily Life in Remitted Patients with Recurrent Depression. A Randomized Controlled Trial. Psychother. Psychosom. 87, 184-186 (2018). 